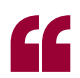

in the presence of electronelectron interactions non-magnetic disorder acts as if it was magnetic to minimize the total energy of the system

\title{
Defects less innocent than they look
}

According to theory, currents flowing along the edges of a $2 \mathrm{D}$ topological insulator should move undisturbed by the omnipresent defects. However, experiments consistently show degraded currents, hinting at a gap in our understanding of the underlying physics. Now, writing in Physical Review Letters, Pietro Novelli and co-workers propose a simple theoretical explanation for these results, linking the current degradation to interaction-induced local magnetism.

Topological materials are a focus of intense research in condensed matter physics, and they hold promise for application in various quantum technologies, such as quantum information and spintronics. In 2D topological insulators, electric currents flow only along the edges of the sample, and electrons with opposite spins travel in opposite directions. The topological properties of the edge currents should protect them from being disturbed by the disorder that is naturally present in any material: theory predicts that only magnetic impurities induce backscattering in the edge states, affecting the electric current.

However, all experimental measurements of conductance in $2 \mathrm{D}$ topological insulators give values that deviate from the theoretical predictions. Experiments performed on different systems, ranging from semiconductor heterostructures to atomically thin crystals, consistently show a current degradation, even in carefully prepared samples in which magnetic impurities are very rare. Several mechanisms have been suggested to explain these observations, but a satisfactory

theoretical explanation has been lacking so far.

The researchers modelled a ribbon of a 2D topological insulator with non-magnetic impurities (vacancies) at the edges. When electron-electron interactions are taken into account, thus going beyond the commonly employed single-particle picture, the model predicts that these non-magnetic defects favour the formation of local magnetic moments. "Technical details aside, there is a very intuitive explanation for this fact: energy minimization," explains Novelli. "In this case, in the presence of electron-electron interactions non-magnetic disorder acts as if it was magnetic to minimize the total energy of the system." This results in the spontaneous breakdown of time-reversal symmetry and in strong backscattering, even at zero temperature. The predicted current degradation at zero temperature, which is observed in experiments, sets this model apart from previous proposed explanations involving electron-electron interactions, in which the disturbances to the current are predicted to vanish at low temperature.

"Interaction-induced magnetism is a phenomenon known to occur around defects in graphene," comments Marco Polini, who coordinated the study. "We thought that in 2D topological insulators the same could happen, causing the degradation of the electric current flow."

Understanding why topological protection is not always effective in real samples could help to design high-quality $2 \mathrm{D}$ topological insulators for devices with a maximized degree of protection. According to the model proposed by Novelli and colleagues, one strategy would be to avoid the presence of short-range non-magnetic (as well as magnetic) impurities in systems with a sizable Coulomb interaction; the alternative would be to produce samples in which the Coulomb interaction is the smallest possible in the first place.

Giulia Pacchioni

ORIGINAL ARTICLE Novelli, P. et al. Failure of conductance quantization in two-dimensional topological insulators due to nonmagnetic

impurities. Phys. Rev. Lett. 122, 016601 (2019) 